\title{
Left Ventricular Pseudoaneurysm Dissecting into the Anterior Chest Wall: A Rare Cause of Sudden Onset Excruciating Chest Pain
}

\author{
Rakshita Chandrashekar ${ }^{1}$, Monoj Kumar Konda ${ }^{2}$, Vishal Gupta ${ }^{2,3}$, Jagadeesh K. Kalavakunta ${ }^{2,3}$ \\ ${ }^{1}$ Department of Cardiology, St John Hospital and Medical Center, Grosse Pointe, MI, USA \\ ${ }^{2}$ Department of Internal Medicine, Western Michigan University School of Medicine, Kalamazoo, MI, USA \\ ${ }^{3}$ Department of Cardiology, Michigan State University/Borgess Medical Center, Kalamazoo, MI, USA
}

\section{Doi: 10.12890/2016_000518- European Journal of Case Reports in Internal Medicine - C EFIM 2016}

\begin{abstract}
Received: $24 / 10 / 2016$
Accepted: 02/11/2016

Published: 00/11/2016
\end{abstract}

\begin{abstract}
How to cite this article: Chandrashekar R, Konda MK, Gupta V, Kalavakunta JK. Left ventricular pseudoaneurysm dissecting int the anterior chest wall: a rare case of sudden onset excruciating chest pain. EJCRIM 2016;3: doi:10.12890/2016_000518.
\end{abstract}

Conflicts of Interests: The Authors declare that there are no competing interests.

This article is licensed under a Commons Attribution Non-Commercial 4.0 License

\section{ABSTRACT}

Left ventricular pseudoaneurysm (LVPA) is associated with a significant mortality rate of up to $45 \%$ in the first year after diagnosis. It is a very rare entity and hence the true incidence and natural history are not clearly known. Clinical presentation varies widely and requires a high index of suspicion for diagnosis. We report the case of a 72-year-old woman with a remote history of left ventricular aneurysm repair during coronary bypass surgery who presented to the emergency department with acute onset of left-sided chest pain and a pulsatile chest wall swelling. She was haemodynamically stable but required an intravenous morphine drip for pain control. Contrast-enhanced computed tomography of the chest showed a large LVPA dissecting through the anterior chest wall. Surgical treatment was discussed with the patient but she opted in favour of comfort care. She died 5 days later from complete rupture of the LVPA. With this report, we aim to raise the level of awareness of LVPA that could anatomically expand and rupture. Early diagnosis and timely surgical intervention is the treatment of choice.

\section{LEARNING POINTS}

- It is important to recognize left ventricular pseudoaneurysm as a rare cause of sudden onset chest pain in a patient with history of myocardial infarction with/without coronary artery bypass grafting and presenting with a pulsatile chest wall mass on physical examination.

- Echocardiography is usually used for diagnosis, which is confirmed by CT or MRI of chest, while open surgical or percutaneous closure are the available treatment options.

- Mortality rates are very high even with surgical treatment in these complex patients.

\section{KEYWORDS}

Left ventricular pseudoaneurysm; acute chest pain; surgical repair of left ventricular aneurysm; computerized tomography in diagnosis of left ventricular aneurysm

\section{CASE DESCRIPTION}

A 72-year-old Caucasian woman with a history of hypertension, myocardial infarction and ischaemic cardiomyopathy underwent coronary artery bypass surgery (CABG). During CABG, she received the left internal mammary artery to the left anterior descending artery, a saphenous vein graft (SVG) to the obtuse marginal and another SVG to the right postero-lateral branch. She also had repair of a small left 
ventricular apical aneurysm with longitudinal incision and excision followed by two-layered longitudinal closure augmented by pledgets. She presented to the emergency department 5 years after repair with sudden onset of excruciating left-sided chest pain, which started while watching television. Physical examination was significant for a pulsatile mass measuring just over $5 \mathrm{~cm}$ over the mitral area (fifth and sixth intercostal spaces). She was normotensive but tachycardic.

\section{METHODS AND PROCEDURES}

Electrocardiogram showed no acute changes. Troponins were elevated at $0.21 \mathrm{ng} / \mathrm{ml}$. Computed tomography (CT) of the chest to evaluate the mass revealed an LVPA measuring $7.4 \times 4.6 \times 7.4 \mathrm{~cm}$ dissecting through the anterior chest wall with a $5.2 \times 2.9 \times 4.6 \mathrm{~cm}$ subcutaneous low density collection in the inferior aspect of the left breast (Fig. 1). The patient required intravenous morphine for pain control. Surgical treatment was discussed with the patient, but she opted against surgery in favour of comfort care at home.

Prior to her presentation, she had been followed up regularly and studies over theyears had showed no pseudoaneurysm. Anechocardiography 3 months before presentation suggested an apical LV aneurysm, which on further evaluation by CT measured $4.8 \times 2.4 \times 7.1 \mathrm{~cm}$ with an appearance highly suggestive of pseudoaneurysm. The patient did not wish to pursue surgical intervention or anticoagulation at the time and had been doing well on medical therapy until the current presentation.

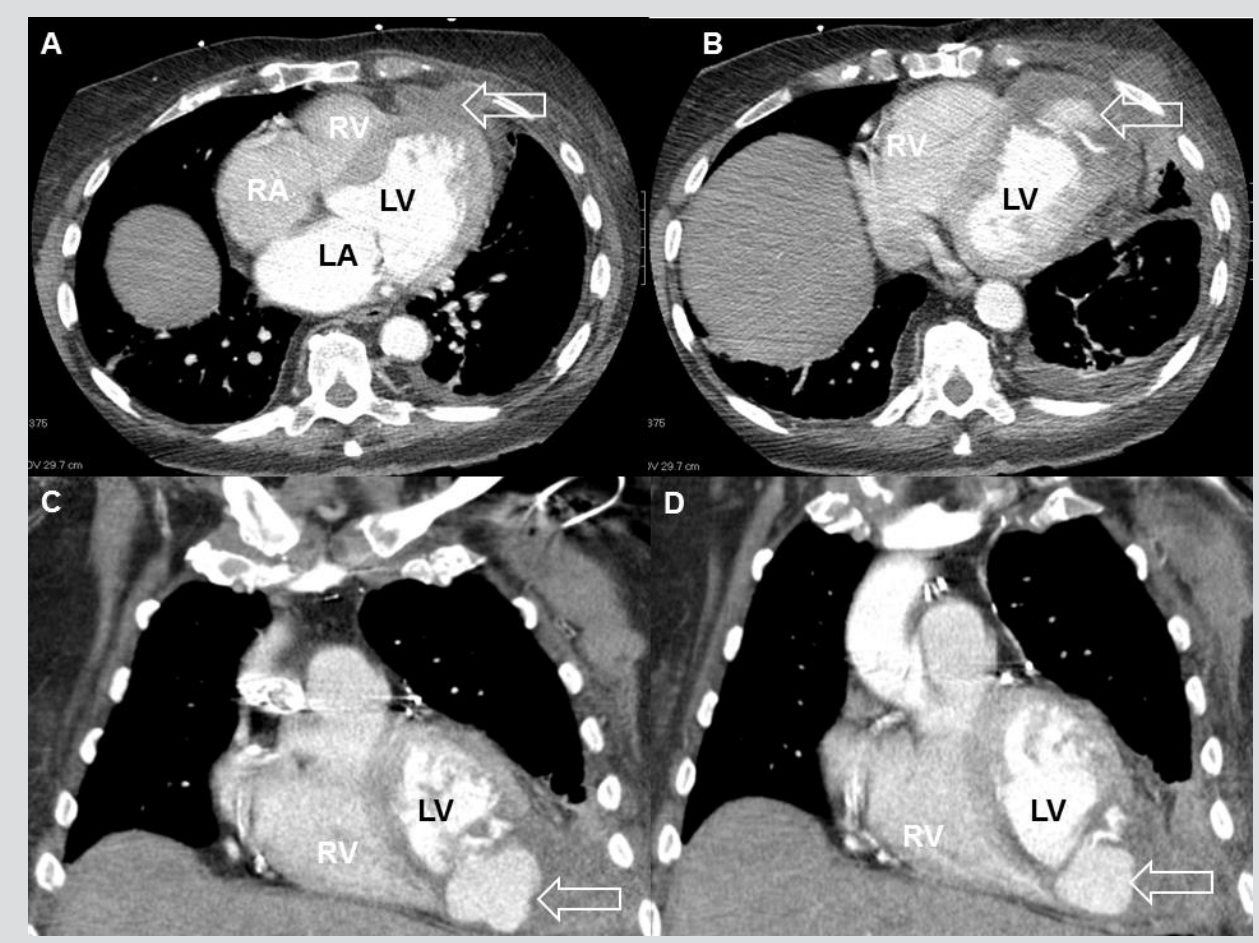

Figure $1(A, B)$ Computed tomography of chest showing a left ventricular pseudoaneurysm (arrow) dissecting through the anterior chest wall via the intercostal condyle. $(C, D)$ Coronal plane images confirming the above findings. $L A$, left atrium; $L V$, left ventricle; $R A$, right atrium; $R V$, right ventricle

\section{DISCUSSION}

Left ventricular pseudoaneurysm (LVPA) is a rare condition that occurs when a ventricular rupture is contained by adherent pericardium, thrombus or scar tissue. A true ventricular aneurysm contains all three layers of the thinned myocardium and often shows systolic bulging motion. A ventricular pseudoaneurysm is characterized by ventricular dilation without all three layers of the ventricle. Usually in pseudoaneurysm, there is a sudden transition of normal myocardium to the aneurysm and it may often contain a thrombus. It is traditionally characterized by a small-neck communication between the left ventricle and aneurysmal cavity (the ratio of the diameter of entry and the maximal diameter of the pseudoaneurysm is $<0.5)$. However, it may have a wide-neck communication ${ }^{[1]}$. Diagnosis and differentiation of true and pseudoaneurysm is critical as the treatment and prognosis differ significantly.

Pseudoaneurysm has more propensity to rupture compared to true aneurysm. According to the law of Laplace, ventricular wall stress (S) is directly proportional to ventricular pressure $(P)$ and ventricular radius $(r)$ and inversely proportional to ventricular wall thickness $(t)$, and is denoted by the formula, $\mathrm{S}=\operatorname{Pr} / 2 \mathrm{t}$. The above three factors, including the thin wall of pseudoaneurysm formed by only a scar tissue, result in 
increased wall stress and a strong tendency to rupture. The true incidence and natural history of pseudoaneurysm is not clearly known as it is a rare phenomenon. Myocardial infarction is the most common cause (>50\%) of pseudoaneurysm. Other causes include LV surgical repair (>33\%), infection (around 5\%) and trauma (>5\%). In our case, the aetiology of the formation of the pseudoaneurysm might be related to either myocardial infarction or the cardiac surgery for LV aneurysm repair. Clinical presentation varies widely: it can present with features of congestive heart failure, arrhythmias, thromboembolic phenomenon or cardiac rupture ${ }^{[2]}$. More than $10 \%$ of cases are asymptomatic and are diagnosed incidentally.

The diagnosis of LVPA is difficult and often the initial presentation is death secondary to cardiac rupture. When suspected, diagnosis can be aided by different imaging modalities. Echocardiography is the most commonly available and utilized modality. Trans-oesophageal threedimensional (3D) echocardiography has improved the diagnostic accuracy. Post-infarction pseudoaneurysms are diagnosed during cardiac catheterization by left ventriculography. When in doubt, LVPA can be confirmed by other modalities such as CT or magnetic resonance imaging (MRI). CT and MRI are also utilized frequently to differentiate pseudoaneurysm from true aneurysm.

Pseudoaneurysms $<3 \mathrm{~cm}$ are usually managed conservatively unless the patient is symptomatic or unstable. The larger the aneurysm, higher is the chance of rupture. Surgical intervention is the mainstay of therapy. Without intervention, pseudoaneurysm has a high risk (up to $45 \%$ ) of rupture within the first year. Even with surgical intervention there is a high mortality rate of up to $35 \%{ }^{[2,3]}$. Surgery involves resection of the pseudoaneurysm and closure of the defect with either suture or a patch. There have been many modifications to the surgical procedure since the original description by Dor (the Dor procedure for left ventricular reconstructive surgery). Transcatheter closure of the pseudoaneurysm is done especially in patients for whom surgery is high risk and who have suitable anatomy for catheter-based intervention ${ }^{[4]}$. Percutaneous closures were achieved with septal occlusion devices, coils and vascular plugs depending on the anatomy ${ }^{[5]}$. Use of multimodality imaging is essential to determine the exact location and the relationship of the pseudoaneurysm with the surrounding structures and will enhance the success of the percutaneous approach. Asymptomatic and elderly patients for whom surgery poses a high risk can be followed up conservatively. In one small case series, patients with mean age of 80 years at the time of diagnosis had a mean survival of $75 \%$ at 4 years of follow-up ${ }^{[6]}$.

\section{REFERENCES}

1. Mele D, Pasanisi G, Capasso F, De Simone A, Morales MA, Poggio D, et al. Left intraventricular myocardial deformation dyssynchrony identifies responders to cardiac resynchronization therapy in patients with heart failure. Eur Heart J 2006;27:1070-1078.

2. Frances C, Romero A, Grady D. Left ventricular pseudoaneurysm. J Am Coll Cardiol 1998;32:557-561.

3. Eren E, Bozbuga N, Toker ME, Keles C, Rabus MB, Yildirim O, et al. Surgical treatment of post-infarction left ventricular pseudoaneurysm: a two-decade experience. Tex Heart Inst J 2007;34:47-51.

4. Narayan RL, Vaishnava P, Goldman ME, Stelzer P, Clark L, Kini AS, et al. Percutaneous closure of left ventricular pseudoaneurysm. Ann Thorac Surg 2012;94:e123-125.

5. Kumar PV, Alli O, Bjarnason H, Hagler DJ, Sundt TM, Rihal CS. Percutaneous therapeutic approaches to closure of cardiac pseudoaneurysms. Catheter Cardiovasc Interv 2012;80:687-699.

6. García-Lledó A, Moya-Mur JL, de Juan J, García-Pérez-Velasco J, Sanz A, Díaz-Caraballo E. Long-term survival of chronic left ventricular pseudoaneurysm in elderly adults. Is surgery the best option? J Am Geriatr Soc 2014;62:2224-2225. 